\section{Forensic Evidence and Crime Scene Investigation}

\section{Introduction}

Contemporary law enforcement has greatly expanded its ability to solve crimes by the adoption of forensic techniques and procedures [1]. Today, crimes often can be solved by detailed examination of the crime scene and analysis of forensic evidence [2]. The work of forensic scientists is not only crucial in criminal investigations and prosecutions, but is also vital in civil litigations, major man-made and natural disasters, and the investigation of global crimes. The success of the analysis of the forensic evidence is based upon a system that emphasizes teamwork, advanced investigative skills and tools (such as GPS positioning, cell phone tracking, video image analysis, artificial intelligence and data mining), and the ability to process a crime scene properly by recognizing, collecting and preserving all relevant physical evidence [3].

Recognition of physical evidence is a vital step in the process. If potential physical evidence is not recognized, collected or properly preserved and tested, the forensic value of the evidence may be greatly reduced or even lost forever. Numerous routine and high profile cases have demonstrated the harsh reality that despite the availability of current crime scene technologies, specialized equipment, and sophisticated forensic laboratory analysis, the effective utilization of physical evidence in crime solving is only as good as the knowledge and integrity of the crime scene personnel ${ }^{1}$ and the objective legal system that supports those functions [4]. In some cases, evidence has been falsified or results tainted, misleading the justice system $[5,6]$.

\section{Scientific Crime Scene Investigation}

Currently, most of the textbooks in the field limit the crime scene investigation to the documentation of the crime scene and the subsequent collection and packaging of the physical evidence. Those who work the crime scene have unfortunately been labeled "crime scene technicians," and often are only involved in the crime scene security, documentation of the crime scene and the collection and preservation of the physical evidence from the crime scene [7]. Documentation of the crime scene and the collection of physical evidence are important aspects of the crime scene investigation and, certainly, must be properly performed. The completion of these crime scene tasks is essential in maintaining the integrity of the physical evidence and providing the final outcome of the criminal investigation. However, these activities are purely mechanical tasks and any individual with minimal training can perform these tasks with moderate precision [8]. While these tasks are extremely important, they have a limited direct value on the solution of a crime.

Scientific crime scene investigation is a process that not only includes the above mechanical aspects of scene security, crime scene documentation, and physical evidence collection and preservation, but also demands and expects more dynamic approaches such as scene survey, scene analysis, development of hypotheses through the linkage of the scene, physical evidence and persons, and the 1 See CSI ON TRIAL where a false confession and faked evidence by a
seasoned crime scene investigator are addressed in a double homicide case in Nebraska. (Available at http://netnebraska.org/basic-page/television/csi-trial-0.)

\section{Journal of} Forensic Investigation
Henry C. Lee ${ }^{1 *}$ and Elaine M. Pagliaro ${ }^{2}$

${ }^{1}$ Distinguished Professor, University of New Haven; Founder, Henry C. Lee Institute of Forensic Science, USA

${ }^{2}$ Henry C. Lee Institute, University of New Haven, USA

Henry C. Lee, Ph.D., Distinguished Professor, University of New Haven, Founder, Henry C. Lee Institute of Forensic Science, USA, Tel: 203-4794594; Fax: 203-931-6074; E-mail: hlee@newhaven.edu

Submission: 10 August 2013

Accepted: 11 September 2013

Published: 13 September 2013
Address for Correspondence

reconstruction of the crime scene [9]. Even in agencies where the crime scene tasks are assigned by levels, all personnel working a crime scene must be aware of the dynamic nature of a crime scene and the importance of linkage and hypothesis development.

Crime scene investigation is based on scientific reasoning. That means that the investigation of the crime scene is systematic, methodical and logical. It begins with the initial response to a crime scene and continues through the scene security, the crime scene documentation, the recognition of physical evidence, the enhancement of patterns, the physical evidence collection, packaging and preservation, the physical evidence examination, the crime scene analysis and profile, and ultimately concludes at the crime scene reconstruction [9]. Furthermore, systematic crime scene investigation is based not only on the principles of the Locard Transfer Theory ${ }^{2}$, but also the Linkage Principle ${ }^{3}$ [9] as shown in Figure 1. The utilization of scientific knowledge in conjunction with the forensic techniques of physical evidence examination will generate investigative leads, and thus ultimately solve the crime.

Physical evidence obtained from the crime scene or other segments of the investigation is often the cornerstone upon which the successful outcome of the case is dependent. Yet, many cases are never solved or prosecuted due to the lack of physical evidence [10]. On the other hand, some innocent people are arrested and prosecuted because of witness misidentification or the misuse of forensic evidence [11]. While it is theoretically possible that a near perfect crime can be committed in which the perpetrator was able to commit the crime, leaving minimal evidence, it is more likely that the critical evidence was never recognized or found. Many investigators have obtained the necessary expertise to document, collect and preserve various types of physical evidence. However, the presumed simple task of finding the critical evidence can often be the failure point of the crime scene investigation.

2 Edmund Locard (1877-1966) was a French criminologist who established the first police crime lab. Locard's Transfer Theory states that the contact between two entities results in a mutual exchange of materials, which can be identified and analyzed by the forensic scientist.

3 Linkage is a connection, relationship or association between objects and /or persons. The "linkage theory explains the interrelations between a crime scene, a victim, a suspect and physical evidence. Understanding and appreciating these connections between these components will provide guidance in determining where evidence may be located and the need to identify evidence so that the linkages may be established." 


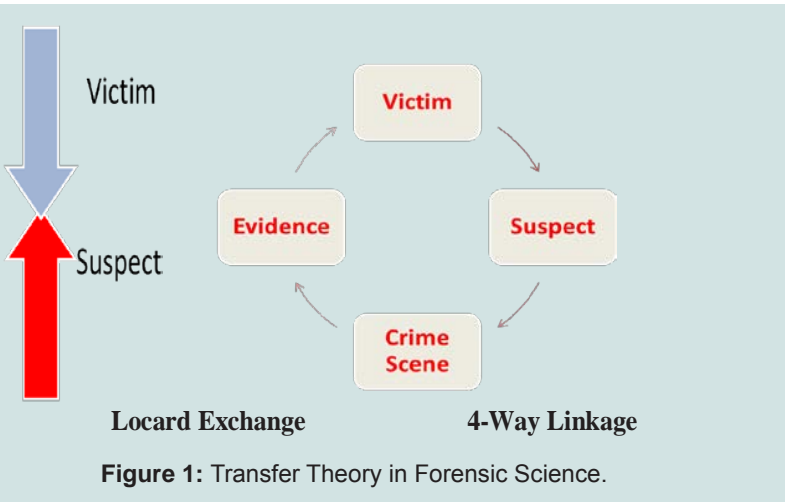

One common practice is to collect everything that could be, in any possible manner, construed as evidentiary in nature [12]. This sort of practice is not only a waste of time and resources, but could potentially cause legal and investigative issues; if every object at a scene is collected and submitted to the forensic laboratory for further analysis, the forensic facility will be overwhelmed, and it is more likely that some of the evidence collected will have no probative value [9]. Examination of materials unrelated to the incident may also provide "false leads" which will waste valuable resources and may lead to the arrest of the wrong person. On the other hand, the identification of evidence that has so-called "probative value" (evidence that is proof of or has a tendency to prove a fact at issue) is sometimes the focus of some crime scene analysts. Care should be taken when using this approach as well, since valuable evidence may be overlooked in an effort to prove a hypothesis or link the scene to a specific suspect. If critical evidence is omitted or improperly preserved, no type of modern, state-of-the-art laboratory instrumentation and technology will be able to salvage the investigation [9]. Hence, a system must be developed where the relevant physical evidence is recognized and located, while superfluous materials are excluded. This will only result if the crime scene investigators understand the goals of a crime scene search, grasp the concepts and techniques for effective searching, and routinely exercise the discipline necessary to rely on a systematic, structured search process with a completely objective and open mind.

After physical evidence has been identified at a scene, subsequent preservation, handling and analysis must satisfy both scientific and legal requirements if that evidence is to be considered reliable and useful in court proceedings.

\section{Types of Crime Scenes}

There are many ways to classify a crime scene [9]:

1. The original location at which the crime was committed (e.g. primary scene, secondary scene);

2. The type of crime committed (e.g., homicide, sexual assault, robbery);

3. The physical location (e.g., indoor, outdoor);

4. The physical condition (e.g., buried, underwater);

5. The boundaries of the scene (e.g., house, train, bank, computer, car);

6. The appearance of the crime scene (e.g. organized, disorganized crime scene);

\section{The activity (e.g. active, passive scenes); and}

8. The size of the crime scene (e.g. universal, macroscopic, microscopic scene).

This appears relatively simple. However, sometimes it is not a clear cut process in a complex situation. While sometimes useful to focus on significant evidence, crime scene personnel should understand these classification systems and how to develop multiple alternate hypotheses based on experience and observation. It is important to keep in mind that there is no single classification method that will satisfy all the elements of the crime scene and its investigation. It is essential that crime scene investigators develop the ability to utilize their analytical skills and logical approaches to make an initial determination regarding the number and types of crime scenes that were involved in the commission of the crime. Finding the exact, precise boundaries of a crime scene may be difficult, and may require adjustments after the initial observations.

Once a crime scene has been defined and a "boundary" has been established, the crime scene(s) should be secured and processed accordingly. Any actions taken at the crime scene should meet all the legal and scientific standards and requirements. Once the locations are determined, the proper sequence of events of the crime can be established, and thus the physical evidence from each of the scenes can be successfully recognized [13]. The crime scene investigation and the forensic process will thus begin.

\section{Physical Evidence at the Crime Scene}

Physical evidence can be classified according to its physical state, the type of crime, and the nature of the evidence, its composition, or the types of questions to be resolved [14]. Each of these classifications is useful for offering conceptual ideas and practical approaches in the crime scene investigation. It is important for a crime scene investigator to understand the value of physical evidence, as well as the limitations of physical evidence, to appreciate the interaction of various definitions or classifications of crime scenes, to recognize the theory of transfer evidence, and to understand the proper methodologies for locating microscopic crime scenes within the more obvious macroscopic crime scenes. It is from the analysis of the crime scene that the crime scene investigator can determine what type of physical evidence will most likely be found at a particular type of crime scene, where to find the physical evidence at a particular type of crime scene, and how to recognize, collect, preserve and process the physical evidence. Finally, based upon the examination results of physical evidence, the investigator should be able to accurately reconstruct the crime scene.

We generally perceive the relevant forensic evidence to include items such as a weapon used at a homicide, a bullet found in a victim's body, or a bloodstain pattern. However, in any given case, any particular object may prove to be the crucial piece of physical evidence necessary to solve the crime. Thus, physical evidence can be best described as any evidence that can provide useful information to investigate the crime [15].

An alternative concept in classification of physical evidence is based upon the nature and form of the evidence: transient, conditional, pattern, transfer, medical, electronic and associative [14].

\section{a. Transient evidence}

Transient evidence is a type of evidence that is, by its very nature, 
temporary, easily changed or lost. Commonly encountered transient evidence includes odors, temperatures, color, and some biological and physical phenomenon such as rigidity or the drying of blood. Due to its temporary nature, this type of evidence must be documented as soon as the evidence is observed.

\section{b. Conditional evidence}

Conditional evidence is generally produced by a set of actions or inactions. Similar to transient evidence, if conditional evidence is not observed and documented immediately at the crime scene, the evidence will be lost forever. Examples of commonly encountered conditional evidence include lighting conditions, television and computer settings, smoke or fire, condition of the victim's body, window positions, or exact locations of specific evidences within the scene.

\section{c. Pattern evidence}

There are a variety of patterns that can be found at a crime scene. Most of these patterns are in the form of imprints, indentations, striations, or other markings such as fractures or depositions. The patterns commonly found at different crime scenes includes blood spatter or stain patterns, glass fracture patterns, fire burn patterns, furniture position patterns, projectile trajectory patterns, track-trail patterns, clothing or article patterns, tire or skid mark patterns, modus operandi patterns, and gun powder or residue patterns.

\section{d. Transfer evidence}

Transfer evidence, also referred to as trace evidence, is generally produced by physical contact between persons, objects, or persons and objects. Some most commonly found transfer evidence is blood, fingerprint, hair, fiber, body fluids, soil, glass, drugs and chemicals. This type of evidence is the traditional forensic evidence examined in the forensic laboratory.

\section{e. Medical evidence}

Medical evidence not only consists of victim's, suspect's, or witness's injuries, the type and degree of injury the location and condition of a wound, the number and size of the wounds, but also includes medical history, the type of medical equipment on the person, house or car, the prescription history, including date and amount prescribed.

\section{f. Electronic evidence}

Due to recent advances in and availability of electronic devices, most individuals own a cell phone, PC, IPod, IPad and other personal devices. Many households and businesses have installed surveillance cameras, monitors and other recording equipment. Investigators should collect and preserve all these types of devices and any digital cameras, phone answering machines, video cameras, computers and data storage devices. Examples of information that may provide investigative leads include cell phone call records, e-mail messages recovered from a suspect's computer hard drive, and videotape recorded from a bank security camera.

\section{g. Associative evidence}

During the course of an investigation, specific items located at a crime scene may be used as evidence to associate a victim or suspect with a particular scene. It may also be used to associate a victim to a suspect. Examples of associative evidence includes suspect's vehicle or wallet found at the crime scene, victim's ring, watch or other personal belongings found on the suspect, receipts, tickets or business cards.

\section{Utilization of Crime Scene Evidence}

Physical evidence recognition and laboratory analysis can be helpful in directing an investigation along a productive path. Not all types of physical evidence will be able to directly link to or identify a suspect. In fact, the most common use of physical evidence found at crime scenes is to identify an unknown substance [16]. Examples include the identification of suspected controlled substances or poisons, identification of accelerants in fire debris, blood alcohol levels, and toxicology of blood specimens. Moreover, physical evidence can provide indirect investigative information or leads to solve a crime. This is the most important and significant use of physical evidence collected from the crime scene.

Not every crime scene will have a "smoking-gun" or an eyewitness, but the scene will definitely contain physical evidence that can provide help to the investigating officers. For example, in a hit-and-run investigation, a seven-layer paint chip from the victim's clothing can be used to narrow down the number and kinds of cars that could have been involved in the accident. Use of this information and vehicle databases may greatly reduce the time spent on the investigation [17]. Furthermore, the size of a shoeprint found at the scene may help the investigator eliminate a suspect or indicate possible shoe class characteristics. The DNA profiles from the semen stain in a victim's rape kit can lead to a potential suspect. The number of different calibers of bullets found may indicate the number and types of weapons used to commit a crime. The locations of blood spatters and fingerprint impressions can yield information about the possible sequence of events at the scene; or the conditions of this pattern evidence could help identify the activities that occurred at the scene.

\section{Features and Process in Forensic Examination}

The objectives of crime scene investigation are to recognize, document, collect, preserve interpret and reconstruct all the relevant physical evidence at the crime scene. The goal is not to predetermine whether a potential suspect is guilty or innocent. The major objective of physical evidence examination is to provide useful information for the criminal investigator to investigate cases. It is the intersection of these objectives of crime scene investigations and the examination of forensic evidence that defines a proper scientific crime scene investigation.

Once the evidence is submitted to the laboratory, scientific examinations will be carried out based on the type of evidence, such as firearms, handwriting, DNA, video, and fingerprint evidence. No matter what type of evidence is being analyzed, laboratory personnel must follow the general principles of evidence examination, which include four independent but connected steps [14].

1. Recognition of an item or pattern as potential evidence or having significance is the first critical step. Failure to recognize crucial evidence will be detrimental to the investigation by failing to establish the vital links between the suspect, victim, crime scene and physical evidence seen in the four-way linkage theory. Successful recognition of evidence depends on the ability to know not only what is present to be found, but 
also where to look for the relevant evidence. The next phase of recognition is the ability to sort through numerous items and objects and to distinguish those with potential or actual relevance from those with no value. Mastery of this skill is only acquired through substantial training and experience.

2. Identification of various forms of evidence is the next logical step. Essentially, identification is a classification scheme. The more characteristics two or more items have in common, the more complex or discriminatory the identification becomes. Within forensic science, items are often identified by comparing selected class characteristics of an unknown object with similar characteristics of a known standard. If the selected class characteristics are essentially the same between the known and unknown samples, then the unknown object can be classified with the known. Moreover, these two samples could have originated from the same source and further analysis is advised. If, however, there are significant differences in some of the selected class characteristics, then the unknown sample can be certainly excluded as coming from the same source as the known standard.

3. Individualization is unique in forensic evidence analysis. It is also the area, which is most criticized and challenged in court. Following identification, forensic scientists may continue with their analysis to determine if a particular sample is unique, even among other members of the same class. This process is referred to as individualization. While not all evidence has sufficient measurable characteristics to be individualized, laboratory examinations will be conducted toward that ultimate goal. If subsequent examinations identify any characteristics that are not common between the known and unknown sample, then the samples are excluded from having the possibility of originating from a common source. Often times, examinations will result in a conclusion that the compared objects are similar in all measurable nature, but they cannot be conclusively linked to a common origin. The recent report of the National Academy of Sciences (NAS) recognized a deficiency and lack of research in this area for some of the forensic disciplines, such as fingerprints and firearms comparisons [4]. On the other hand, a statistical analysis of the significance of the common features may be helpful in evaluating the significance of individualization. In some disciplines, matches of characteristics will generally be subject to statistical analysis, as with DNA profiles. These statistical interpretations are only as reliable as the populations and databases from which the conclusions are deduced [18]. Finally, not all types of forensic evidence can be individualized; nor is there any statistical data associated with information stored in the some databases.

4. Reconstruction is the final phase in the forensic examination process. This phase is entirely dependant upon proper recognition, identification, individualization and interpretation of relevant evidence. Reconstruction utilizes investigative information, crime scene information, and laboratory analysis of the physical and pattern evidence. The reconstruction process has been defined as one that involves the use of both inductive and deductive logic [9]. This can be a complex task in which many types of physical evidence, pattern information, analytical results, investigative information, and other documentary and testimonial evidence are linked into one entity. The amount of information that a reconstruction may provide is limited by the above factors. The more relevant and accurate the data compiled in a particular case, the greater the chance that reconstruction exercises will be of value to the investigation [19].

Crime scene reconstruction as a process is not limited to the recognition, collection and preservation of evidence. It also requires the determination or elimination of events or actions that occurred at the crime scene through the analysis of the crime scene patterns, the location, sequence of deposit and position of the physical evidence, and the laboratory examination results of the physical evidence. Crime scene reconstruction involves the scientific analysis of a scene or the interpretation of the scene pattern evidence; it involves the systematic study of related information ending in the logical formulation of a theory. The process of forensic examination is presented in Figure 2.

\section{Crime Scene as a Component of the Forensic System}

Crime scene investigation, as noted previously, is not just processing or documenting a crime scene, nor is it just the collection or packaging of physical evidence. Careful processing, documentation and collection of physical evidence are integral parts of the forensic processes. But the foundation of all forensic investigations is the ability of the crime scene investigator or forensic scientist to recognize potential physical evidence - large or small, visible or latent, exculpatory or inculpatory - at a crime scene. The subsequent identification of the physical evidence along with the determination of the possible source or origin of that evidence is the next step. Proper crime scene investigation is the starting point in the process of establishing what happened, when it happened, where it happened, who was involved, and how it occurred.

Contrary to the way it is portrayed in popular television programs, crime scene investigation is neither glamorous nor carried out in an easy or lackadaisical manner. It is an organized, methodical, systematic and logical process. To perform crime scene functions properly requires not only extensive training and experience on the part of the investigator, but also absolute objectivity along with a coordinated team effort. Perhaps one of the most important issues related to crime scene investigation today is the establishment of professional standards [4]. An assessment of current standards in the

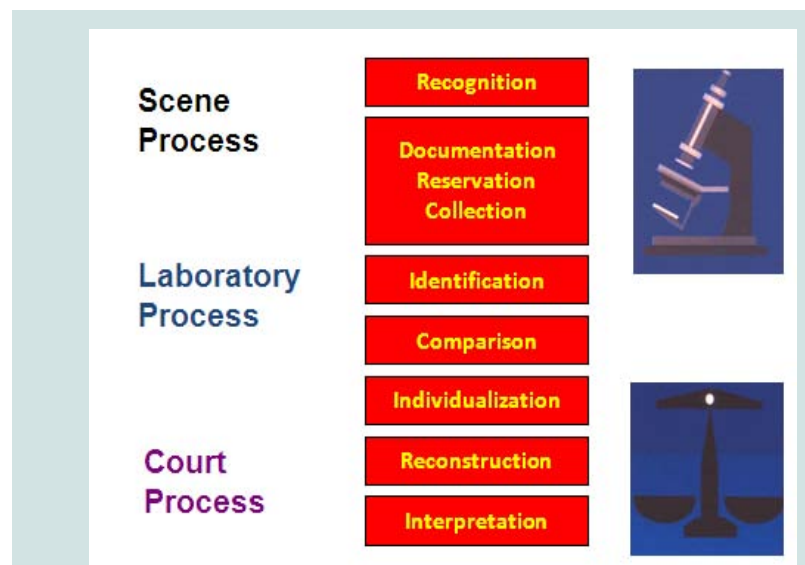

Figure 2: The Forensic Examination Process. 
practices of collection, examination, and analysis of physical evidence is needed. All professionals involved with crime scene investigation, forensic examination and the legal system must carefully assess their roles in the investigative process and presentation of the forensic evidence in courts of law. Professionalism and ethical standards for all parties involved in both criminal and civil litigations need to be developed.

Despite the countless historical cases from which we can learn and improve, beginning with the OJ Simpson case, we continue to witness serious crimes going unsolved or ending without justice being served because of the needless errors associated with poor crime scene investigation practices. Virtually no jurisdiction in the United States or any foreign county has resolved this dilemma. It is ironic that while forensic science has experienced significant technological advancements and improvements, the forensic profession is more highly scrutinized, and the integrity of forensic scientists is often challenged in court. If the crime scene is not properly managed, all the technology and advancements we currently possess in the forensic laboratory become worthless. If forensic evidence is not introduced during a court preceding or wrongfully presented, the judicial system's ability to serve justice is diminished.

A high-quality crime scene process does not have to be elaborate or sophisticated. Rather, the simple, objective adherence to fundamental principles and procedures is all that is necessary. The essential crime scene functions are recognition, documentation, collection and preservation of all relevant physical evidence. If these elements are achieved, there will be a much higher probability that the case will be accurately resolved through scientific interpretation of the evidence and presentation of the available facts and data in the court system.

\section{Conclusion}

It is the police officers, detectives, crime scene investigators or evidence technicians that usually complete the crime scene search and begin the forensic investigation stages. Crime scene investigators and laboratory personnel continue the forensic analysis of evidence to obtain data that can be useful to the justice system. During the pretrial or initial litigation stages, the prosecution and defense counsel determine which physical evidence will be utilized. During the trial or adjudication stages, the judge determines the admissibility of the forensic evidence. There is no guarantee that any of these parties who are part of the evidentiary process will sufficiently understand the full potential of forensic evidence. This understanding of practitioners can be enhanced through continued education at conferences, workshops and other training opportunities.

After two years of study, the NAS issued a final report on the status of forensic science. As highlighted in that report, several areas require attention to improve forensic science: The need for empirical research in some forensic subspecialties; the need for certification, accreditation and quality assurance/ quality control programs in the forensic laboratory; the importance of increasing funding for laboratories; education needs for scientist; and standardization [4]. The training, education and standardization of crime scene investigation are equally critical for the improvement of forensic services. This training can take many forms including traditional classroom and workshop formats, access to on-line programs, webinars and degree programs in investigation or forensic science. As a greater number of police officers and attorneys acquire updated information and receive new, specialized training in forensic science, the current situation should improve. The end result would be to make forensic science even more valuable and to maintain the high quality of justice that this society deserves.

\section{References}

1. Inman K, Rudin N. (2001) p 41 in Principle and Practice of Crimnalistics: the profession of forensic science. CRC Press, Boca Raton, FL.

2. Joseph L. Peterson, John P. Ryan, Pauline J Houlden, Steven ihajlovic (1986) Forensic Science and the Courts: The Uses and Effects of Scientific Evidence in Criminal Case Processing, NCJRS Reports. U.S. Department of Justice, Washington, DC.

3. Gaensslen, RE, Harris H, Lee, HC (2008) Introduction to Forensic Science and Criminalistics. Pp 61-79. McGraw-Hill, New York.

4. Committee on Identifying the Needs of the Forensic Science Community (2009) Strengthening Forensic Science In The United States: A Path Forward. The National Academies Press, Washington DC.

5. Pyrek, KM (2007) Forensic Science under Siege: The Challenge of Forensic Laboratories and the Medico-Legal Investigation System. Elseiver Press, Burlington, MA

6. Moriarty, JC, Saks, MJ (2005) Forensic Science: Grand Goals, Tragic Flaws, and Judicial Gatekeeping. Judges' Journal 44 : 16-33.

7. National Medicolegal Review Panel (2011) Death Investigation: A Guide for the Scene Investigator (Technical Update). US Department of Justice, Office of Justice Programs, Washington, DC.

8. Hawthorne, M. (1999) First Unit Responder: A Guide to Physical Evidence Collection for Patrol Officers. CRC Press, Boca Raton, FL.

9. Lee, HC, Palmbach, TM, Miller, M (2001) Henry Lee's Crime Scene Handbook. Academic Press, San Diego, CA.

10. Truman, JL, Planty M. (2012) Criminal Victimization in the United States, 2011. Bureau of Justice Statistics Office of Justice Programs, Washington, DC.

11. Gianelli, P (2007) Wrongful Convictions and Forensic Science: The Need to Regulate Crime Labs. (North Carolina Law Review 86: 163- 187.

12. Fisher, B, Fisher, D (2012) Techniques of Crime Scene Investigation. CRC Press, Boca Raton, FL.

13. Technical Working Group on Crime Scene Investigation (2000) Crime Scene Investigation: A Guide for Law Enforcement. US Department of Justice Office of Justice Programs, Washington DC.

14. Lee, HC, Harris, H (2011) Physical Evidence in Forensic Science. Lawyers and Judges Publishing Co., Inc, Phoenix, AZ.

15. Lee, HC, Palmbach, TM (2006) Crime Scene Management. Pp 577 - 593 in Forensic Science and Law (ed. by Cyril Wecht and John Rago). CRC Press, Boca Raton, FL.

16. Peterson, J (2005) Census of Publicly Funded Forensic Crime Laboratories, 2002. Bureau of Justice Statistics Bulletin (NCJS 207205, 2005). US Department of Justice US Bureau of Justice Statistics, Washington, DC.

17. Bowen, R,Schnieder J (2007) Forensic Databases: Paint, shoeprints and beyond. NIJ Journal $258: 34-38$.

18. Aitken,CGG (2004) Populations and Samples. Pp 51-83 in the Use of Statistics in Forensic Science (ed. by C.G.G. Aitken and David A. Stoney) Taylor \& Francis, London, UK.

19. Miller, M (2009) Crime Scene Investigation (3rd ed.). Pp 167-188 in Forensic Science: An Introduction to Scientific and Investigative Techniques (ed. by Stuart James and Jon Nordby) CRC Press, Boca Raton, FL. 